\title{
Archéopages
}

Archéopages

Archéologie et société

Hors-série 1 | 2008

Construction $^{s}$ de l'archéologie

\section{Les souterrains aménagés, un sujet d'archéologie préventive}

\section{Marc Viré}

\section{(2) OpenEdition}

1 Journals

Édition électronique

URL : https://journals.openedition.org/archeopages/842

DOI : 10.4000/archeopages.842

ISSN : 2269-9872

Éditeur

INRAP - Institut national de recherches archéologiques préventives

Édition imprimée

Date de publication : 1 février 2008

Pagination : $39-40$

ISSN : 1622-8545

\section{Référence électronique}

Marc Viré, "Les souterrains aménagés, un sujet d'archéologie préventive », Archéopages [En ligne],

Hors-série 1 | 2008, mis en ligne le 01 février 2008, consulté le 24 février 2023. URL : http://

journals.openedition.org/archeopages/842 ; DOI : https://doi.org/10.4000/archeopages.842

Tous droits réservés 
Ces derniers rassemblèrent leurs guerriers en les contraignant à résider au pied de leur château. L'émergence d'une agglomération autour du château attira alors de nombreux artisans et marchands. C'est ainsi que se développa peu à peu une jôkamachi, une «ville-au-pieddu-château »; en d'autres termes, une ville castrale seigneuriale. L'archipel s'est alors rapidement urbanisé avec des cités construites autour d'un château seigneurial surmonté d'un donjon. Ce fut le cas d'Edo (Tôkyô), Ôsaka, Nagoya ou encore Kanazawa, villes seigneuriales construites autour de la résidence fortifiée d'un suzerain.

Il y a là une forte ressemblance avec le processus de construction des châteaux d'Europe occidentale durant le Moyen Âge. En Europe comme au Japon, le développement d'une classe de guerriers, s'appuyant sur une solide féodalité, a donné naissance, selon des modalités assez proches, à des formes architecturales et matérielles relativement similaires.

\section{Les souterrains aménagés, un sujet d'archéologie préventive}

Marc Viré

Inrap, UMR 8589, Lamop

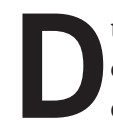
u mythe à la réalité. Le mythe des souterrains est largement répandu en France. Il existe une solide croyance autour des souterrains de liaison allant de tel à tel village, de tel château à telle abbaye. Lors de la construction du Tgv Sud-Est, les habitants de Blandy-lès-Tours, en Seine-et-Marne, surveillaient nuit et jour les terrassements, guettant la découverte du souterrain qui était présumé relier le château à la tour de l'Aiguillon. Hélas, à leur grande déception, rien de tel ne fut trouvé. Même à Paris, la croyance en l'existence de souterrains anciens sous la Seine a la vie dure, et les recherches en ce domaine suscitent toujours quelque déception en démontrant qu'il n'en existe pas.

Pourtant, notre territoire est parsemé de cavités creusées par l'homme, qu'il s'agisse de carrières, de marnières, de caves en réseau, de souterrains aménagés ou encore d'habitats troglodytiques. Les carrières (Guini-Skliar et al. 200o), lieux industriels et préindustriels, sont à traiter à part. En revanche, la question des habitats troglodytiques est plus proche de nos interrogations sur les souterrains, en particulier lorsqu'ils sont, tel ceux de Haute-Isle dans le Val-d'Oise, bien datés de l'époque carolingienne (Viré 1932). Les cavités dont il est ici question sont de plan et d'organisation multiples et le plus souvent d'étendue restreinte. Elles sont désignées par le terme vague mais commode de «souterrain aménagé». Quand elles sont plus étendues comme pour les «muches» de Picardie (Petit 2001), leur dimension atteint facilement
200 mètres dans la plus grande longueur. Les recherches portant sur ces cavités sont anciennes et ont été traitées, d'une manière fondatrice, par Adrien Blanchet (1923) au début du $\mathrm{Xx}^{\mathrm{e}}$ siècle.

De la description factuelle à l'interdisciplinarité. Il existe une typologie bien établie des différents types de cavités. Des descriptions précises ont été faites par de nombreuses sociétés savantes, qui ont traité le sujet dans le cadre de congrès de «subterranologie ». La gamme des travaux est ample, allant des publications les plus savantes aux articles et livres véhiculant les fantasmes les plus loufoques.

D'une manière générale, la difficulté d'une approche scientifique repose sur le fait de pouvoir distinguer entre l'usage des lieux et l'intention d'origine. Qu'a voulu faire celui qui a creusé ? L'utilisation de la cavité correspondelle à celle qui a prévalu lors de sa création ? Quelles directions donner à la recherche? Deux voies sont possibles: soit une approche descriptive avec une analyse minutieuse de l'organisation de la cavité, soit une démarche globalisante et interdisciplinaire.

La première approche est celle des sociétés savantes spécialisées: elle donne une part importante à la description minutieuse des éléments (salles, couloirs sinueux, trou de visée ou d'aérage, silos), en négligeant la surface. J'ai entendu dire, autrefois, dans le Périgord que «les souterrains aménagés étaient creusés au milieu de nulle part», niant par là la simple possibilité que des habitats associés en surface aient pu disparaître.

La seconde approche a été tentée par des archéologues professionnels (Gady 1989; Viré 1983), qui ont compris que le rapprochement avec l'habitat de surface était important, et l'archéologie préventive y a largement apporté sa contribution.

La construction de l'autoroute A19 entre Orléans et Courtenay a été l'occasion de nombreuses opérations d'archéologie préventive confiées à l'Inrap. Dans le Loiret, près d'Aschères-le-Marché, un souterrain en contexte avec un habitat médiéval a enfin été découvert (Gillotte 2007). Au regard du plan d'occupation du sol, l'implantation de l'accès au sein du hameau montre bien une relation entre l'habitat et le souterrain aménagé. Si la fouille appelle les remarques habituelles (les passages difficiles pour l'accès aux salles, entre autres), elle en convoque beaucoup d'autres. Finalement, pourquoi un souterrain plutôt qu'un silo enterré ou construit? Ces lieux devaient-ils également servir à cacher les habitants? La question reste posée quant à la signification du geste fondateur.

Une approche complémentaire est fournie par l'étude des «muches» de Picardie (Petit 2001). Elle repose sur des prospections avec relevés topographiques, études des lieux très détaillées et soin attentif porté à la collecte d'objets et de graffitis. La documentation et l'analyse 
montrent que les muches ont été utilisées comme cachettes par les populations pendant la guerre de Trente Ans. Mais, de l'aveu même de Bernard Petit, la démonstration de cette utilisation ne résout pas la question du «pourquoi » des différents creusements. Il signale cependant, avec justesse, une attribution possible d'une salle à chaque famille du village, appuyée en cela par la trouvaille de nombreuses serrures et clés.

Des pistes plus larges paraissent devoir être suivies, comme l'analyse de la structure géologique du sous-sol de la région. Il faut d'abord rechercher si les terres du proche sous-sol sont propices au creusement de cavités, sous le village en particulier. Les terres lourdes ou argileuses conviennent mal au creusement de silos depuis le sol, et encore moins à celui de caves sous les maisons. Les muches paraissent suppléer à cette nécessité : elles sont systématiquement creusées dans la craie, matériau permettant le drainage des eaux. Certes, dans la Somme, le substrat est essentiellement constitué de ce matériau, mais il faudrait se pencher sur la structure géographique et géologique de chaque village. Un petit talweg, avec quelques alluvions, peut facilement avoir conduit à abandonner le creusement d'une cave. Dans le Limousin, l'étude de plusieurs cas a montré qu'une infime minorité de souterrains aménagés se trouvent situés à moins de 100 mètres d'un talweg.

La composante sociologique. Une seconde piste serait l'analyse de la structure de la population et du rapport à la terre. La question est de savoir si un usage collectif des terres était alors en vigueur. Ces éléments sont bien connus des géographes qui ont étudié la population bretonne des monts d'Arrée: il existe dans la région de Carhaix une forte tradition collective liée à la pauvreté de la terre, opposée à la configuration sociale de la riche plaine du bas Léon, entre Landivisiau, Saint-Pol-de-Léon et Ploudalmézeau. À Carhaix, cette tradition «collectiviste» est encore visible aujourd'hui dans l'implantation du vote « rouge» lors des élections, alors que le nord du Finistère connaît un vote «bleu» tout aussi bien établi.

Le rapport peut ne pas sembler évident, mais il est indispensable d'intégrer ces pistes de réflexion à l'étude des maisons et des villages médiévaux. Les recherches portant sur la structure des silos, des divers bâtiments et des stockages ont beaucoup progressé ces dernières années. Nombre d'études de cas et leur mise en série débouchent peu à peu sur une géographie des usages, et il paraît important de savoir pourquoi tel groupe de maisons ne dispose pas de cavités souterraines alors que tel autre en possède. Nous pourrions ainsi commencer à saisir les différentes dimensions des muches collectives de Picardie, par exemple: chacun avait sa cave dans un ensemble creusé par et pour le village, là où il était possible de creuser. Ce type de souterrain collectif résulterait donc autant d'un choix d'organisation sociale que de facteurs géologiques. Se pose également une question majeure : celle de la répartition entre habitat groupé et habitat dispersé, car nous savons désormais que cette distinction a pu être beaucoup plus mouvante que nous ne l'imaginions.

Le croisement de divers registres de compréhension se révèle aujourd'hui indispensable. Il est donc temps de remettre dans l'analyse générale un élément qui en avait été exclu ou, du moins, fortement marginalisé : le monde souterrain. Nous voyons ici se profiler un constat récurrent: la distinction entre la création des choses et leur usage. Les pistes ont trop souvent été brouillées par la combinaison des deux. On ne creuse pas un souterrain en temps de guerre pour se cacher ; le souterrain a été creusé en d'autres temps ou en d'autres circonstances. Le fait de s'y cacher serait alors purement conjoncturel. Or, c'est justement le fait de séjourner qui produit des traces archéologiques, voilà la difficulté. Nous ne pratiquons pas encore l'archéologie des intentions, mais, si nous devions y être conduits, nous aurions à combiner sciences humaines et sciences exactes. On est précisément là au cœur du métier d'archéologue. Le milieu souterrain est une occasion d'interdisciplinarité, tout à la fois déroutante et passionnante.

Blanchet A. 1923: Les Souterrains-refuges de la France, Paris, A. et J. Picard, 1923 (réédition, 1983).

GADY S. 1989: Les Souterrains médiévaux du Limousin, Paris, Éd. de la Maison des sciences de l'homme (DAF 19).

GiLlotTE S. 2007: Aschères-le-Marché, DFs de fouille préventive, Inrap, 2007.

Guini-Skliar A., Viré M., Lorenz J., Gély J.-P., BlanC A. 2000 Les Souterrains de Paris, les anciennes carrières souterraines, Douai, Nord Patrimoine Éditions.

Petit B. 2001: «Les «muches `, une résidence rurale collective durant les XVI ${ }^{\mathrm{e}}$ et XVII ${ }^{\mathrm{e}}$ siècles. Étude des souterrains aménagés du canton de Villers-Bocage (Somme) ", Revue archéologique de Picardie, 2001, $\mathrm{n}^{\circ} 1 / 2$, p. $73-125$

VIré A. 1932: Le Village troglodytique de Haute-Isle (Seine-et-Oise), Société préhistorique française, Publication de la Commission des souterrains et excavations artificielles de la France.

VIRÉ M. 1983: Carriers et carrières au terroir de Saint-Jean-de-Latran à Paris, XII ${ }^{e}-X V I^{e}$ siècles. Thèse de doctorat, université Paris I. 